\title{
Fractal-based radiomic approach to predict complete pathological response after chemo-radiotherapy in rectal cancer
}

Citation for published version (APA):

Cusumano, D., Dinapoli, N., Boldrini, L., Chiloiro, G., Gatta, R., Masciocchi, C., Lenkowicz, J., Casa, C., Damiani, A., Azario, L., Van Soest, J., Dekker, A., Lambin, P., De Spirito, M., \& Valentini, V. (2018). Fractal-based radiomic approach to predict complete pathological response after chemo-radiotherapy in rectal cancer. Radiologia medica, 123(4), 286-295. https://doi.org/10.1007/s11547-017-0838-3

\section{Document status and date:}

Published: 01/04/2018

DOI:

10.1007/s11547-017-0838-3

\section{Document Version:}

Publisher's PDF, also known as Version of record

\section{Document license:}

Taverne

Please check the document version of this publication:

- A submitted manuscript is the version of the article upon submission and before peer-review. There can be important differences between the submitted version and the official published version of record.

People interested in the research are advised to contact the author for the final version of the publication, or visit the DOI to the publisher's website.

- The final author version and the galley proof are versions of the publication after peer review.

- The final published version features the final layout of the paper including the volume, issue and page numbers.

Link to publication

\footnotetext{
General rights rights.

- You may freely distribute the URL identifying the publication in the public portal. please follow below link for the End User Agreement:

www.umlib.nl/taverne-license

Take down policy

If you believe that this document breaches copyright please contact us at:

repository@maastrichtuniversity.nl

providing details and we will investigate your claim.
}

Copyright and moral rights for the publications made accessible in the public portal are retained by the authors and/or other copyright owners and it is a condition of accessing publications that users recognise and abide by the legal requirements associated with these

- Users may download and print one copy of any publication from the public portal for the purpose of private study or research.

- You may not further distribute the material or use it for any profit-making activity or commercial gain

If the publication is distributed under the terms of Article $25 \mathrm{fa}$ of the Dutch Copyright Act, indicated by the "Taverne" license above, 


\title{
Fractal-based radiomic approach to predict complete pathological response after chemo-radiotherapy in rectal cancer
}

\author{
Davide Cusumano ${ }^{1,2} \cdot$ Nicola Dinapoli $^{3} \cdot$ Luca Boldrini $^{2} \cdot$ Giuditta Chiloiro $^{2} \cdot$ Roberto Gatta $^{3} \cdot$ Carlotta Masciocchi $^{2}$. \\ Jacopo Lenkowicz ${ }^{2} \cdot$ Calogero Casà $^{3} \cdot$ Andrea Damiani $^{3} \cdot$ Luigi Azario $^{4} \cdot$ Johan Van Soest $^{5} \cdot$ Andre Dekker $^{5}$. \\ Philippe Lambin ${ }^{5} \cdot$ Marco De Spirito $^{4} \cdot$ Vincenzo Valentini $^{2}$
}

Received: 18 September 2017 / Accepted: 14 November 2017 / Published online: 11 December 2017

(c) Italian Society of Medical Radiology 2017

\begin{abstract}
The aim of this study was to propose a methodology to investigate the tumour heterogeneity and evaluate its ability to predict pathologically complete response (pCR) after chemo-radiotherapy (CRT) in locally advanced rectal cancer (LARC). This approach consisted in normalising the pixel intensities of the tumour and identifying the different sub-regions using an intensity-based thresholding. The spatial organisation of these subpopulations was quantified using the fractal dimension (FD). This approach was implemented in a radiomic workflow and applied to $198 \mathrm{~T} 2$-weighted pre-treatment magnetic resonance (MR) images of LARC patients. Three types of features were extracted from the gross tumour volume (GTV): morphological, statistical and fractal features. Feature selection was performed using the Wilcoxon test and a logistic regression model was calculated to predict the pCR probability after CRT. The model was elaborated considering the patients treated in two institutions: Fondazione Policlinico Universitario "Agostino Gemelli" of Rome (173 cases, training set) and University Medical Centre of Maastricht ( 25 cases, validation set). The results obtained showed that the fractal parameters of the subpopulations have the highest performance in predicting $\mathrm{pCR}$. The predictive model elaborated had an area under the curve (AUC) equal to $0.77 \pm 0.07$. The model reliability was confirmed by the validation set (AUC $=0.79 \pm 0.09$ ). This study suggests that the fractal analysis can play an important role in radiomics, providing valuable information not only about the GTV structure, but also about its inner subpopulations.
\end{abstract}

Keywords Radiomics $\cdot$ Fractals $\cdot$ Rectal cancer $\cdot$ Predictive model $\cdot$ Magnetic resonance imaging

\section{Introduction}

The use of biomedical imaging for diagnosis and therapy purposes in oncology has exponentially increased in the last few decades. The interpretation of these images is crucial to perform correct diagnosis and to choose the most appropriate treatment for the patient [1].

Radiomics is an emerging discipline that aims to analyse biomedical imaging through a quantitative approach, allowing the detection of physio-pathological features of the

Electronic supplementary material The online version of this article (https://doi.org/10.1007/s11547-017-0838-3) contains supplementary material, which is available to authorized users.

Giuditta Chiloiro

vchiloiro@gmail.com

Extended author information available on the last page of the article healthy or tumour tissues that is not directly estimable by visual inspection $[2,3]$.

To date, radiomics has been applied to several tumours (lung, breast and prostate [4-6]) and for different diagnostic imaging techniques (computed tomography (CT), magnetic resonance (MR), and positron emission tomography (PET) $[4,7,8])$, showing promising results.

The high number of parameters extractable from digital images, together with clinical and pathological patient data, can be correlated with clinical outcomes to elaborate clinical decision support tools [9].

This work focused on the application of radiomics on MR images of patients affected by locally advanced rectal cancer (LARC).

The standard care for these patients is represented by neoadjuvant chemo radiation therapy (CRT) followed by total mesorectal excision (TME) [10, 11]. Approximately 11-42\% of these patients show a pathological complete response 
(pCR) after CRT; different studies demonstrated that patients showing pCR usually have a better prognosis in terms of local failure, metastases-free survival and overall survival $[12,13]$. Recent studies have proposed more conservative surgical approaches, such as local excision or wait and see, to reduce morbidities related to TME in patients, in which complete response is observed $[14,15]$.

Elaborating predictive models that are able to identify which patients could result to be clinical complete responders therefore gained great importance for their correct multidisciplinary management.

The model developed in this investigation aims to predict the $\mathrm{pCR}$ probability analysing pre-treatment T2-weighted MR images. Magnetic Resonance Imaging (MRI) represents the gold standard in rectal cancer diagnosis, as it provides excellent soft tissue contrast and high spatial resolution [16].

Because of these characteristics, this imaging modality can describe with high precision the heterogeneity of the tumour, which is considered today as an important biomarker for response prediction. Recent studies have demonstrated that tumour sensitivity to treatments could be linked to tumoral spatial organisation, which reflects its heterogeneous cellular population [17].

Fractal Analysis is considered a reliable method to quantify the tumour heterogeneity [18]. Fractals are structures that display a repeating pattern at different size scales; this property is quantified by a parameter named fractal dimension that measures the self-similarity grade of the structure under analysis [19]. An image processing procedure that combines fractal and segmentation analysis has been proposed to investigate cancer heterogeneity on MR images, similar to the approach described by Szigeti et al., for studying lung tumour heterogeneity on mice CT scans [20].

We first identified different tumour subpopulations through an intensity-based segmentation applied on normalised values and then their spatial organisation has been described using fractal dimensions. The aim of this study was to implement our fractal-based approach in a radiomic analysis to quantify the rectal cancer heterogeneity in patients affected by LARC and to elaborate a predictive model able to esteem the pCR probability of a patient by analysing the pre-treatment MR.

\section{Materials and methods}

\section{Patients}

This study was conducted on the pre-treatment MR images of the patients treated in two hospital centres: Fondazione Policlinico Universitario "Agostino Gemelli" (Rome, Italy) and MAASTRO Clinic (GROW, MUMC, Maastricht, the Netherlands).
Policlinico Gemelli retrospectively collected 173 patients among those treated between May 2008 and December 2014. MAASTRO Clinic prospectively collected 25 patients considering the THUNDER trial (THeragnostic Utilities for Neoplastic DisEases of the Rectum, NCT 00969657).

The enrolled patients had a pathological diagnosis of LARC and they were older than 18 years, when the disease was diagnosed. All the patients signed informed consent for data collection and they were treated with neo-adjuvant chemo-radiotherapy followed by total mesorectal excision 6-8 weeks after the end of CRT.

The pathological post-operative data included histology, grading and tumour regression grade (TRG) according to Mandard classification [21]. Pathological complete response was defined in case of ypT0N0 or ypN0/ypNx.

\section{Image analysis}

The MR images analysed were obtained following a protocol that considers T2-weighted fast spin-echo 2D images acquired in a plane orthogonal to the tumour longitudinal axis [16]. No intravenous contrast agents were administered. Further details about image acquisition parameters are available as supplementary material.

The MR images were then uploaded on a radiotherapy delineation console (Eclipse, Varian Medical System ${ }^{\mathrm{TM}}$, Palo Alto, California, USA) and the gross tumour volume (GTV) was delineated by a team of two radiation oncologists and two radiologist experts in rectal cancer, following the guidelines defined in ICRU n.83 [22].

The DICOM files containing the MR images and the corresponding RT Structure files were imported in Moddicom, an open source R (R Core Team, Vienna, Austria) package [23] developed by the KBO Labs of the Fondazione Policlinico Universitario "Agostino Gemelli" to perform quantitative image analysis [24].

Figure 1 shows the image processing applied to each case to analyse tumour heterogeneity, studying the spatial organisation of the subpopulations inside it. It consisted of three steps:

\section{Normalisation}

Considering the pixel intensities $P(\mathrm{x}, \mathrm{y})$ of a determined GTV structure, they were normalised according to the following formula:

$$
N(x, y)=\frac{P(x, y)-\min [I(x, y)]}{\max [I(x, y)]-\min [I(x, y)]},
$$



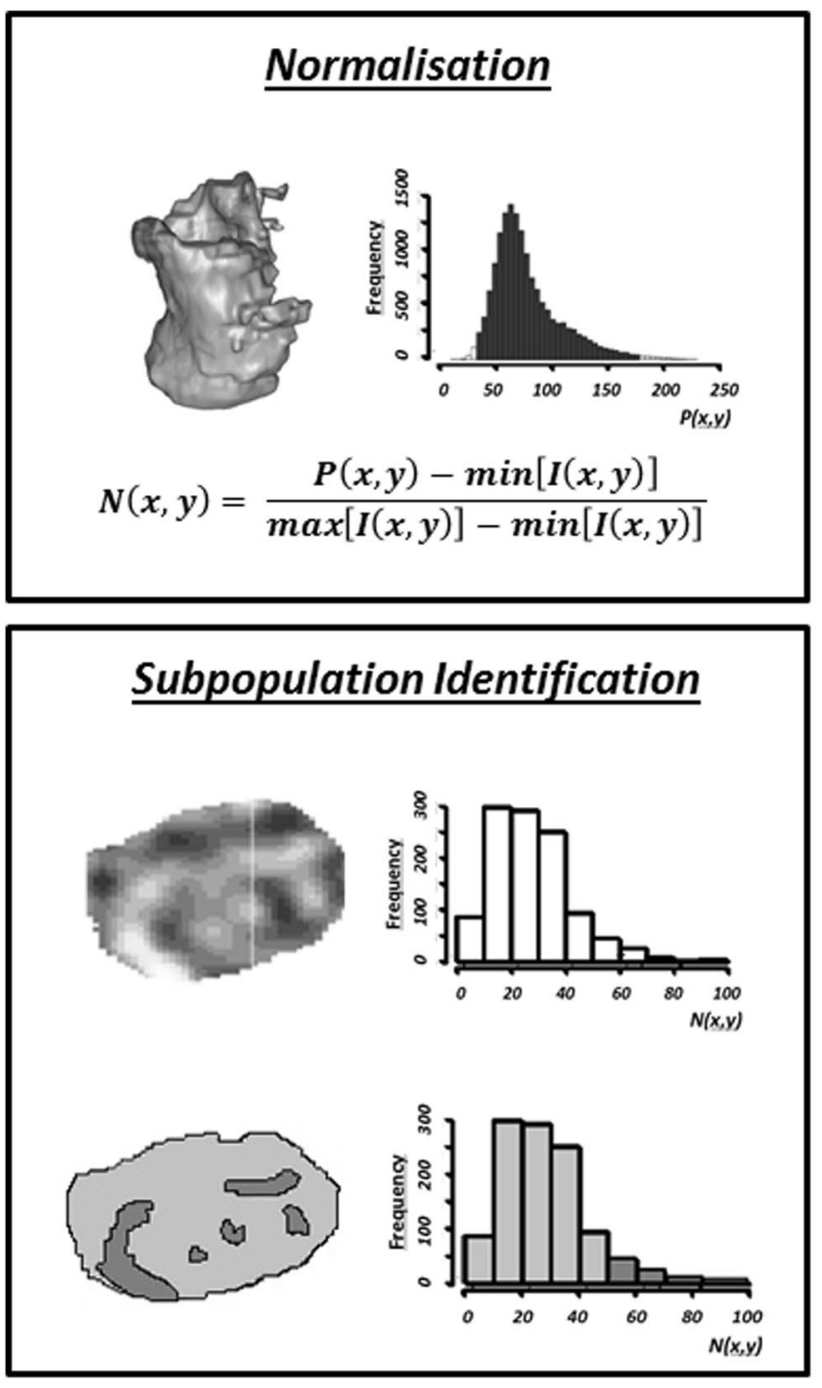

Fig. 1 Schematic representation of the image processing, developed to analyse tumour heterogeneity in a patient. The original pixel values contained inside the GTV volume were normalised considering the 1 st and 99th percentile of the grey-level histograms. Different pixel

where $\min [I(x, y)]$ and $\max [I(x, y)]$ are, respectively, the 1st and the 99th percentile of the grey-level histograms representing the GTV, to minimise the influence linked to the spike pixels.

\section{Subpopulations identification}

Different clusters were identified in the GTV slices containing the normalised intensity values. The segmentation was automatically performed considering the pixels whose intensities were included between two threshold levels defined as percentages of the GTV maximum. Pixels with

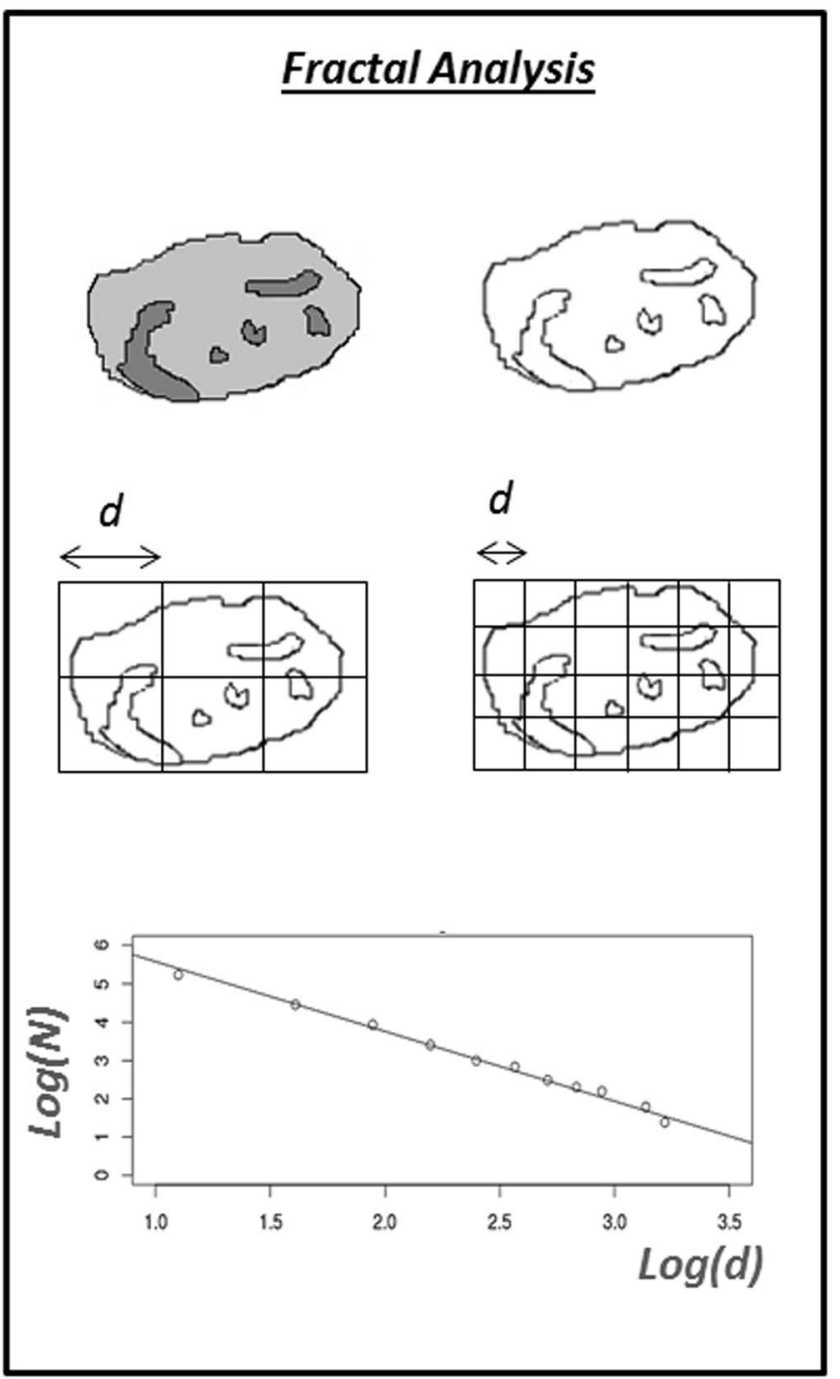

clusters (subpopulations) were identified considering various intensity thresholds. The box-counting method was applied slice by slice to estimate the fractal dimension of the subpopulations identified

similar intensity levels were gathered and considered as new structures, here defined as "subpopulations".

\section{Fractal analysis}

The spatial organisation of these subpopulations was quantified, calculating the fractal dimension of the border including the analysed subpopulation.

The fractal dimension was calculated using the software developed in $\mathrm{R}$ and $\mathrm{C}$ environment implementing the boxcounting method. This procedure consisted in covering the image with fixed-side (d) squares and counting the number $(N)$ of squares containing the image border. This process was then repeated with the change of the side square size. The 
number of counts as a function of the side square was plotted on a log-log graph and a linear fit is applied. The angular coefficient of the linear fit was an estimation of the fractal dimension [25].

This software was validated comparing its results with those obtained using the ImageJ software [26] on simple geometries with known fractal dimensions (line, square, Koch curve).

For each individuated subpopulation, the FD calculation was performed on all GTV slices; mean, median, maximum and minimum FD distribution values were considered as parameters representing the spatial organisation of the subpopulation.

\section{Feature extraction}

Three types of features were automatically calculated by means of Moddicom:

(1) Fractal features, considered as parameters quantifying the tumour heterogeneity.

(2) Morphological features, describing the tumour geometry and GTV shape (surface and volume, surface/volume ratio, eccentricity).

(3) Statistical features, describing the behaviour of greylevel histogram representing the GTV volume (entropy, skewness and kurtosis).

The statistical features were calculated applying the Laplacian of Gaussian (LoG) filter to the raw images.

This filter consists of an implemented convolution kernel that applies the following equation to the original pixels:

$\operatorname{LOG}(x, y)=\frac{1}{\pi \sigma^{2}}\left[1-\frac{x^{2}+y^{2}}{2 \sigma^{2}}\right] e^{-\frac{x^{2}+y^{2}}{2 \sigma^{2}}}$

where $x$ and $y$ represent the coordinates of pixels surrounding the central one (on which equation is used to calculate the convolution) and $\sigma$ is the size of standard deviation in the LoG equation, expressed in $\mathrm{mm}$ [27].

This filter, adopted by $\mathrm{Ng}$ et al. [28]. on CT images of colorectal cancer, was used to smooth the high-frequency noise of the images and enhance the variation of values among adjacent pixels. Different $\sigma$ values were investigated, ranging from 0.2 to $4 \mathrm{~mm}$.

No filter was instead used on the subpopulations individuated for the fractal analysis, according to the different works calculating FD on original images [29, 30].

\section{Data analysis}

All the extracted features were collected in a database and associated to the clinical TNM classification at diagnosis and pathological outcome data (pCR versus not $\mathrm{pCR}$ ).
A predictive model was then elaborated having patients enrolled by the Policlinico Gemelli as training set (TS) and those from MAASTRO Clinic as validation set (VS).

The heterogeneity between the training and validation cohorts was evaluated in terms of Wilcoxon-Mann-Whitney and Pearson's $\chi^{2}$ test [31]. Highly relevant features were identified as a first step for data analysis using the Wilcoxon test method.

This test was recently addressed as the most reliable and accurate method for feature selection in radiomics by Parmar et al. [32], who compared 14 feature selection methods used in different radiomic studies. Features were considered significant, when $p$ value was lower than 0.05 . The features selected by this test were then collected in a cross-correlation matrix, to identify and eliminate redundant features [31].

Many linear logistic regression models were elaborated to predict the binary outcome (pCR vs. no $\mathrm{pCR}$ ) using the features that showed low mutual correlation values in the cross-correlation matrix $(|R|<0.3)$.

The best predictive model was selected using the Akaike information criteria [33].

The discrimination power of the final model was evaluated in terms of Receiver Operating Characteristic (ROC) analysis, and an external validation was performed using the MAASTRO patients as validation set [34].

A nomogram was then produced for a visual representation of the predictive model.

\section{Results}

Table 1 summarises the clinical characteristics of the patients involved in this study. The statistical tests performed confirmed the heterogeneity of the cohorts adopted.

Pathologic complete response $(\mathrm{TRG}=1)$ was reached in 47 patients of Rome (pCR occurrence rate equal to 27\%) and in 7 patients of Maastricht (pCR occurrence rate equal to $28 \%$ ). The proportions observed between positive and negative outcomes were consistent with clinical literature data [12].

The majority of the Rome patients (83.3\%) had a prescription dose of $55 \mathrm{~Gy}$. The same dose has been prescribed to all the Maastricht patients. No significant relationship between radiotherapy dose and $\mathrm{pCR}$ probability has been observed.

Table 2 contains the results of the Wilcoxon test obtained for the fractal features; most of these features showed high significance in separating pCR and no pCR patient groups.

The median fractal dimension calculated on the GTV original contours (as manually delineated by the radiation oncologists) showed a $p$ value equal to 0.008 (subpopulation 0-100 in Table 2). 
Table 1 Patient's characteristics and descriptive statistics of variables in the cohort patients of Policlinico Gemelli (Rome, 173 patients) and MAASTRO Clinic (Maastricht, 25 patients)

\begin{tabular}{|c|c|c|c|c|c|c|}
\hline & \multirow{2}{*}{\multicolumn{2}{|c|}{$\frac{\text { Rome }}{\text { Patient's characteristics }}$}} & \multicolumn{2}{|c|}{ Maastricht } & \multicolumn{2}{|c|}{$p$ values for differences } \\
\hline & & & & & & \\
\hline & \multicolumn{4}{|l|}{ Age } & \multicolumn{2}{|c|}{ Statistic test } \\
\hline & Mean & Range & Mean & Range & $\chi^{2}$ test & MW test \\
\hline \multicolumn{7}{|l|}{ Sex } \\
\hline Male & 64 & $28-84$ & 63.3 & $52-80$ & - & 0.573 \\
\hline Female & 61.9 & $43-80$ & 63.8 & $47-73$ & - & 0.698 \\
\hline Age & 63.0 & $28-84$ & 63.5 & $47-80$ & - & 0.878 \\
\hline \multicolumn{7}{|c|}{ Clinical features } \\
\hline & $N$ & $\%$ & $N$ & $\%$ & $\chi^{2}$ test & MW test \\
\hline \multicolumn{7}{|l|}{ Stage } \\
\hline \multicolumn{7}{|l|}{$\mathrm{cT}$} \\
\hline 2 & 15 & 8.7 & 1 & 4 & 1.105 & - \\
\hline 3 & 100 & 57.8 & 20 & 80 & & \\
\hline 4 & 58 & 33.5 & 4 & 16 & & \\
\hline \multicolumn{7}{|l|}{$\mathrm{cN}$} \\
\hline 0 & 10 & 5.8 & 3 & 12 & 0.455 & - \\
\hline 1 & 60 & 34.7 & 7 & 28 & & \\
\hline 2 & 103 & 59.5 & 15 & 60 & & \\
\hline \multicolumn{7}{|l|}{ Response } \\
\hline $\mathrm{TRG}=1$ & 47 & 27 & 7 & 28 & 1.000 & - \\
\hline TRG $>1$ & 126 & 73 & 18 & 72 & & \\
\hline \multicolumn{7}{|l|}{ RT dose } \\
\hline$<50.4$ & 7 & 4 & - & - & - & - \\
\hline 50.4 & 19 & 11 & - & - & - & - \\
\hline 55 & 144 & 83.3 & 25 & 100 & - & - \\
\hline$>55$ & 3 & 1.7 & - & - & - & - \\
\hline \multicolumn{7}{|c|}{ Concurrent CT } \\
\hline Yes & 169 & 97.7 & 25 & 100 & - & - \\
\hline No & 4 & 2.3 & - & - & - & - \\
\hline
\end{tabular}

In the last few columns, the statistical tests to investigate the statistical significance are reported $\chi^{2}$ test pearson's $\chi^{2}$ test, $M W$ test Mann-Whitney test, $N$ number, $c T$ clinical T stage, $c N$ clinical $N$ stage, $T R G=1$ pathological complete response, $T R G>1$ pathological not complete response, $R T$ radiotherapy, $C T$ chemotherapy
The median FD evaluated for the 0-60 subpopulation and the maximum FD for the 40-100 and 50-100 subpopulations showed higher statistical significance $(p$ value $=0.001)$ in identifying pCR patients in respect to median FD on the original GTV contours.

The morphological features did not show statistical significance in separating pCR and no pCR patient groups.

For the statistical features, the highest significance was observed for entropy $(p=0.048)$ and skewness $(p=0.006)$, when the LoG filter was applied to MR images $(\sigma=0.34 \mathrm{~mm}$ for entropy, $0.48 \mathrm{~mm}$ for skewness).

The cross-correlation matrix containing the significant features is available in the supplementary material.

The fractal and statistical features were uncorrelated overall (maximum absolute correlation value $|R|=0.47$, median absolute correlation value $|R|=0.21$ ). Entropy and skewness showed $|R|<0.1$ for all the sigma sizes of the applied LOG filter.

The best predictive model resulting from the Aikake analysis took into account two clinical features identified at diagnosis ( $\mathrm{cT}$ and $\mathrm{cN}$ ) and three parameters extracted from the MR staging images:

- The skewness calculated applying a LoG filter with $\sigma=0.48 \mathrm{~mm}$;

- The entropy calculated applying a LoG filter with $\sigma=0.34 \mathrm{~mm}$;

- The maximum FD calculated on the subpopulation constituted by pixels with intensities between 40 and 100\% of GTV maximum. 
Table $2 p$ values obtained by applying the Wilcoxon-Mann-Whitney test to evaluate $\mathrm{pCR}$ prediction ability for the different fractal features investigated to varying the subpopulations

\begin{tabular}{lllll}
\hline Threshold & Mean FD & Median FD & Min FD & Max FD \\
\hline $0-20$ & 0,405 & 0.333 & 0.625 & 0.051 \\
$0-40$ & 0.003 & 0.002 & 0.152 & 0.004 \\
$0-60$ & 0.001 & 0.002 & 0.022 & 0.003 \\
$0-80$ & 0.002 & 0.004 & 0.017 & 0.078 \\
$0-100$ & 0.008 & 0.008 & 0.100 & 0.160 \\
$10-30$ & 0.061 & 0.068 & 0.408 & 0.019 \\
$10-50$ & 0.008 & 0.006 & 0.150 & 0.016 \\
$10-70$ & 0.022 & 0.014 & 0.374 & 0.040 \\
$20-40$ & 0.043 & 0.023 & 0.460 & 0.023 \\
$20-60$ & 0.032 & 0.018 & 0.261 & 0.042 \\
$20-80$ & 0.066 & 0.037 & 0.516 & 0.029 \\
$30-50$ & 0.025 & 0.017 & 0.805 & 0.009 \\
$30-70$ & 0.035 & 0.017 & 0.944 & 0.003 \\
$30-90$ & 0.030 & 0.012 & 0.794 & 0.010 \\
$40-60$ & 0.011 & 0.011 & 0.490 & 0.006 \\
$40-80$ & 0.012 & 0.015 & 0.306 & 0.003 \\
$30-100$ & 0.034 & 0.017 & 0.923 & 0.012 \\
$40-100$ & 0.011 & 0.010 & 0.487 & 0.001 \\
$50-100$ & 0.007 & 0.015 & 0.236 & 0.001 \\
$60-100$ & 0.014 & 0.022 & 0.268 & 0.006 \\
$70-100$ & 0.129 & 0.092 & 0.572 & 0.154 \\
\hline
\end{tabular}

Table 3 Covariates and coefficients of the linear logistic regression model elaborated to predict pCR starting from the analysis of $\mathrm{T} 2$ weighted staging MR images

\begin{tabular}{lrll}
\hline Covariate & Coefficient & $\sigma$ (coefficient) & $p$ value \\
\hline Intercept & 11.366 & 6.119 & 0.063 \\
cT & -0.997 & 0.3772 & 0.007 \\
cN & 0.619 & 0.359 & 0.084 \\
Skewness $(\sigma=0.48 \mathrm{~mm})$ & -3.601 & 1.361 & 0.008 \\
Entropy $(\sigma=0.34 \mathrm{~mm})$ & 2.948 & 1.719 & 0.086 \\
Max FD $(40-100)$ & -9.862 & 3.228 & 0.002 \\
\hline
\end{tabular}

The values of the parameters and coefficients characterizing the model are reported in Table 3.

Figure 2 shows the ROC curves of the model developed on training (left) and validation set (right); the AUC values obtained were 0.775 and 0.790 , respectively.

Figure 3 shows a nomogram of the model, which includes all the considered covariates.

\section{Discussion}

\section{Radiomics in MRI: difficulties and opportunities}

The application of radiomics to MRI had minor diffusion in comparison to CT and PET applications, even if MRI today represents the most accurate imaging technique for soft tissues.

This limited diffusion is correlated to the high complexity of the MR images process and standardization. Signal intensity can indeed be highly variable in these images, as it results from a complex interplay of different factors, joining tissue properties (such as relaxation time), patient specific characteristics and technical scan acquisition parameters [3].

Although several methods have been proposed to overcome the standardization problem (such as histogram matching or normalisation to an external ROI signal), the individuation of a common strategy is still far [35].

McGarry et al. [7] have recently proposed a strategy to normalise the MR signal in brain, dividing each voxel intensity by the standard deviation of the whole brain signal.

This methodology was applied to the MR images of 81 glioblastoma patients and a predictive model was then proposed to identify patients with poor prognosis at the time of tumour diagnosis. In particular, the authors quantified tumour heterogeneity, creating different radiomic profiles originating from the combination of the information extracted from four different MR contrasts.

In this investigation, we presented a new approach to process the MR signal, consisting in the normalisation of each pixel inside the GTV in reference to the 1st and 99th percentile of the overall GTV intensity level histogram, to eliminate spike signals and focus on the informative content of the analysed GTV.

\section{Potentialities of fractal analysis in radiomics}

After the normalisation, we evaluated the tumour heterogeneity analysing the spatial organisation of the pixel clusters with common intensities by means of fractal dimensions, as performed by Szigeti et al., on lung cancer CT images [20].

The results obtained in the feature selection process showed that the fractal parameters related to tumour subpopulations have higher performance in predicting $\mathrm{pCR}$ than statistical and morphological features.

The high informative content of the fractal dimension was also confirmed in the elaboration of the predictive model, where the maximum FD of the subpopulation with pixel intensity higher than $40 \%$ appeared to be the most significant parameter of the model. 

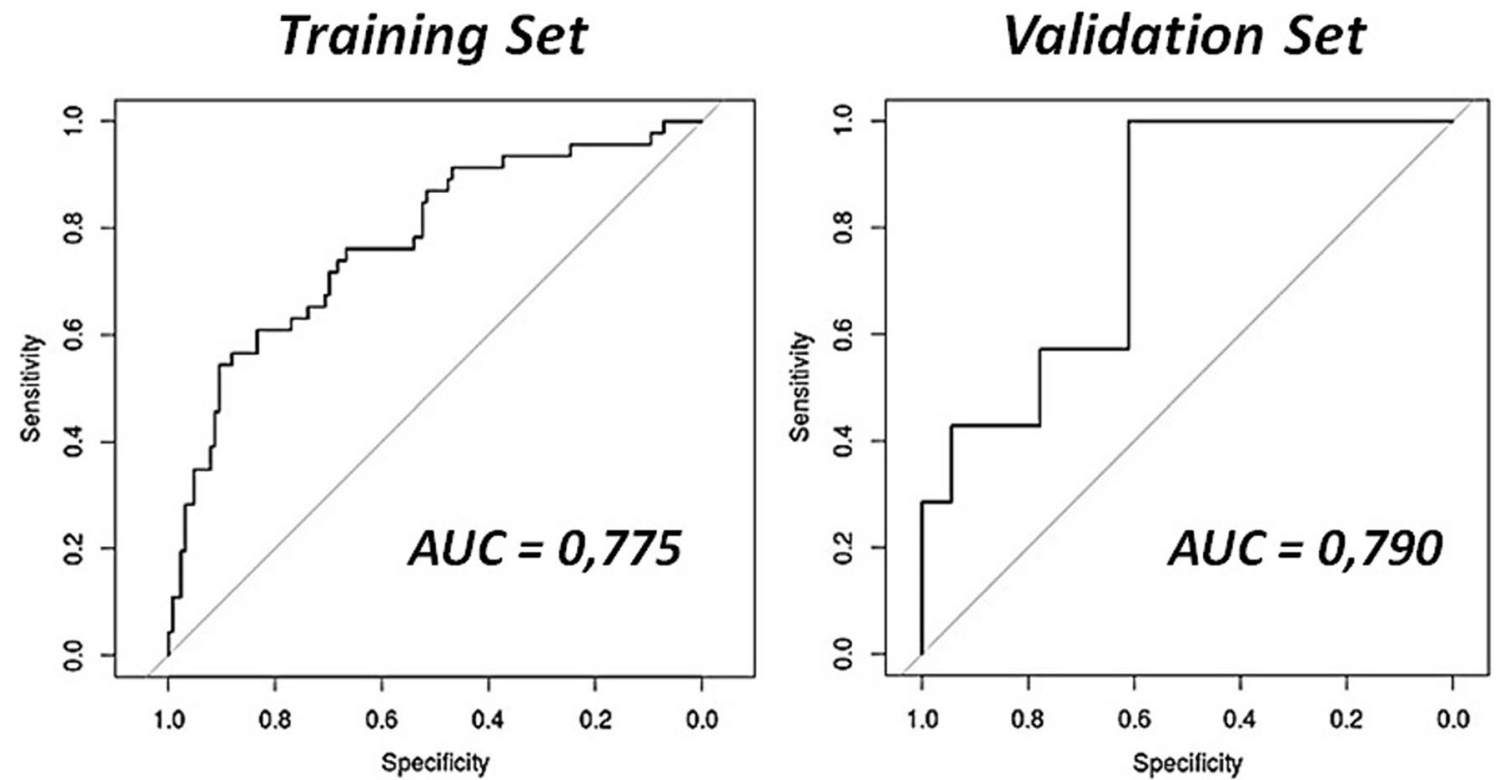

Fig. 2 ROC curves and AUC values representing the performance of the predictive model elaborated calculated for the training (left) and validation set (right)

Points

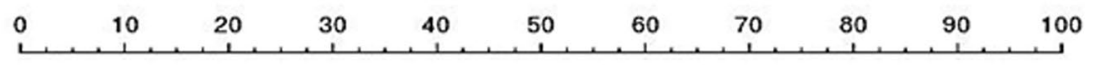

$c T$

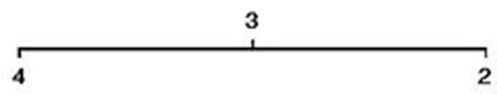

$c N$

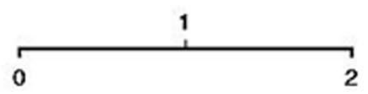

Skewness

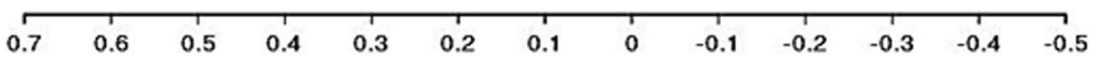

Entropy

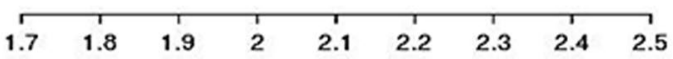

$\operatorname{Max} F D(40-100)$

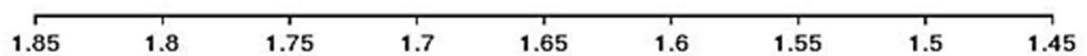

Total Points

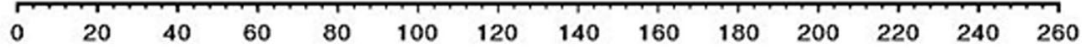

pCR Probability

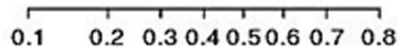

Fig. 3 Nomogram of the predictive model developed 
The proposed statistical model predicts with high accuracy $(\mathrm{AUC}=0.78$ in training set and 0.80 in validation set) whether pCR will be obtained for a specific patient, starting from the analysis of the staging MR.

A predictive model for pCR starting from 18FDG PET-CT images was recently proposed by van Stiphout et al. [36]. The model, elaborated on 190 patients, showed predictive performances comparable to our model in training set $(\mathrm{AUC}=0.78)$ but lower in the validation set $(\mathrm{AUC}=0.70)$.

In 2015, Intven et al. [37] proposed a model to evaluate treatment response after CRT in LARC patients, by analysing different MR sequences (T2w volumetry, DW-MRI and DCE-MRI) acquired before and after CRT. In their explorative study (55 analysed patients), they observed that a significant increase in the apparent diffusion coefficient (ADC) after CRT was correlated to non-responder patients.

One of the most significant advantages offered by our model is represented by the fact that only MR images acquired before treatment are required to calculate the pCR probability. In this way, the model can be useful in clinical practice to evaluate the best chemo-radiotherapy treatment to offer to the specific patient, moving towards a vision of personalised medicine.

\section{New frontiers in fractal-based radiomics}

One of the limitations of our model is that the fractal analysis was performed only in 2D modality; this choice is linked to the different pixel resolutions between the planar image and the slice thickness.

The implementation of 3D fractal analysis, by means of interpolation algorithms and mesh-based approaches, would be interested to investigate the self-similarity of the entire disease volume.

However, the fact that a parameter as the maximum FD, related to a single tumour slice and obtained by a $2 \mathrm{D}$ analysis, provides the most valuable information in the predictive model reflects the idea that the overall tumour aggressiveness can be determined by the characteristics of a single part of the disease.

In particular, we observed that a higher FD value is predictive of a lower pCR probability, as it reflects a more complex and compact tumour structure (as shown in Fig. 4).

This effect could be explained, supposing that pixels with intensity higher than $40 \%$ correspond to the areas of the tumour with a specific metabolic hallmark (e.g., hypoxia). Further studies are required to verify this or other interesting hypotheses at a cellular level (e.g., angiogenesis, vascularization, receptor expression), by comparing MR images with pathological specimen data and imaging biomarkers information.
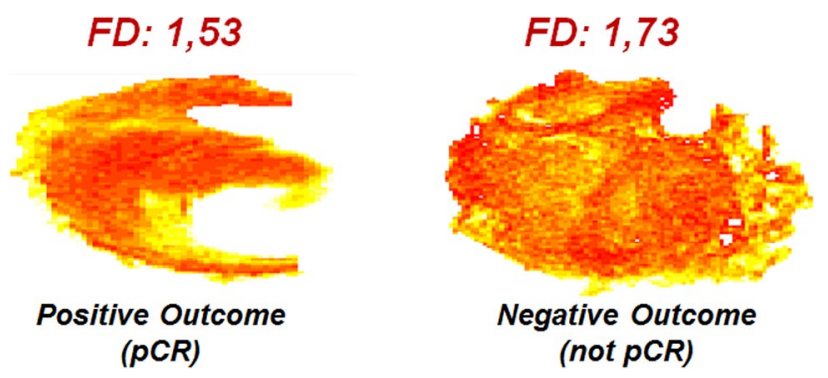

Fig. 4 GTV slice where the maximum FD was calculated for a patient who experimented pCR (left) and for a patient who did not show pCR (right).In the red scales, the pixel values with normalised intensities higher than $40 \%$ are reported

\section{Conclusion}

The possibility to provide spatial information related to tumour sub-regions can furthermore open important scenarios in biophysics and radiotherapy planning. In fact, assuming that the pixel clusters with shared intensity levels correspond to cell groups with individual physical and radiobiological characteristics, the possibility to detect these clusters could allow dose modulation within the tumour through dose-painting protocols.

In conclusion, the method proposed in this work is able to detect from MR images, valuable information about the rectal tumour heterogeneity, to predict the $\mathrm{pCR}$ probability after CRT in case of LARC patients.

Funding This study was not funded by any company.

\section{Compliance with ethical standards}

Conflict of interest All authors declare that they have no conflict of interest.

Ethical approval All procedures performed in studies involving human participants were in accordance with the ethical standards of the institutional and national research committee and with the 1964 Helsinki declaration and its later amendments or comparable ethical standards. Informed consent was obtained from all the individual participants.

\section{References}

1. Gillies R, Kinahan P, Hricak H (2016) Radiomics: images are more than pictures, they are data. Radiology 278:2

2. Lambin P, Rios-Velazquez E, Leijenaar R et al (2012) Radiomics: extracting more information from medical images using advanced feature analysis. Eur J Cancer 48:441-446

3. Kumar V, Gu Y, Basu S et al (2012) Radiomics: the process and the challenges. Magn Reson Imaging 30(9):1234-1248 
4. Coroller TP, Grossmann P, Hou Y et al (2015) CT-based radiomic signature predicts distant metastasis in lung adenocarcinoma. Radiother Oncol 114(3):345-350

5. Chan A, Tuszynski JA (2016) Automatic prediction of tumour malignancy in breast cancer with fractal dimension. R Soc Opensci 3:160558

6. Waliszewski P (2016) The Quantitative criteria based on the fractal dimensions, entropy, and lacunarity for the spatial distribution of cancer cell nuclei enable identification of low or high aggressive prostate carcinomas. Front Physiol 7:34

7. McGarry S, Hurrell S, Kaczmarowski A et al (2016) Magnetic resonance imaging-based radiomic profiles predict patient prognosis in newly diagnosed glioblastoma before therapy. Tomography 2(3):223-228

8. Cook G, Siddique M, Taylor B et al (2014) Radiomics in PET: principles and applications. Clin Transl Imaging 2:269-276

9. Aerts HJ, Velazquez ER, Leijenaar RT et al (2014) Decoding tumour phenotype by noninvasive imaging using a quantitative radiomics approach. Nat Commun 5:40

10. Sauer R, Liersch T, Merkel S et al (2012) Preoperative versus postoperative chemoradiotherapy for locally advanced rectal cancer: results of the German CAO/ARO/AIO-94 randomized phase III trial after a median follow-up of 11 years. J Clin Oncol 30:1926-1933

11. Valentini V, van Stiphout RG, Lammering G et al (2015) Selection of appropriate end-points (pCR vs 2yDFS) for tailoring treatments with prediction models in locally advanced rectal cancer. Radiother Oncol 114:302-309

12. Vecchio FM, Valentini V, Minsky BD et al (2005) The relationship of pathologic tumor regression grade (TRG) and outcomes after preoperative therapy in rectal cancer. Int J Radiat Oncol Biol Phys 62:752-760

13. Macchia G, Gambacorta MA, Masciocchi C et al (2017) Time to surgery and pathologic complete response after neoadjuvant chemoradiation in rectal cancer: a population study on 2094 patients. Clin Transl Radiat Oncol 4:8-14

14. O'Neill BD, Brown G, Heald RJ et al (2007) Non-operative treatment after neoadjuvant chemoradiotherapy for rectal cancer. Lancet Oncol 8:625-633

15. Maas M, Beets-Tan RG, Lambregts DM et al (2011) Wait-and see policy for clinical complete responders after chemoradiation for rectal cancer. J Clin Oncol 29:4633-4640

16. Barbaro B, Fiorucci C, Tebala C et al (2009) Locally advanced rectal cancer: MR imaging in prediction of response after preoperative chemotherapy and radiation therapy. Radiology 250:730-739

17. Vogelstein B, Papadopoulos N, Velculescu VE et al (2013) Cancer genome landscapes. Science 339:1546-1558

18. Alic L, Niessen WJ, Veenland JF (2014) Quantification of heterogeneity as a biomarker in tumor imaging: a systematic review. PLoS ONE 9:e110300

19. Mandelbrot B (1982) The fractal geometry of nature. Ed Brickmann, J Free Co, San Francisco
20. Szigeti K, Szabó T, Korom C et al (2016) Radiomics-based differentiation of lung disease models generated by polluted air based on X-ray computed tomography data. BMC Med Imaging 16:14

21. Mandard AM, Dalibard F, Mandard JC et al (1994) Pathologic assessment of tumor regression after preoperative chemoradiotherapy of esophageal carcinoma. Cancer 3(11):2680-2686

22. International Commissioning on Radiation Units and Measurements. (2010) prescribing, recording, and reporting intensitymodulated photon-beam therapy (IMRT).ICRU Report 83

23. R Core Team (2014). R: a language and environment for statistical computing. Vienna, Austria: R Foundation for Statistical Computing; https://www.r-project.org

24. Dinapoli N, Alitto AR, Vallati M et al (2015) Moddicom: a complete and easily accessible library for prognostic evaluations relying on image features. Conf Proc IEEE Eng Med Biol Soc. https:// doi.org/10.1109/EMBC.2015.7318476

25. Smith TG, Lange GD, Marks WB (1996) Fractal methods and results in cellular morphology-dimensions, lacunarity and multifractals. J Neurosci Method 69(2):123-136

26. Rasband WS. ImageJ. Version 7.0. Bethesda (MD): U. S. National Institutes of Health USA; 1997-2016

27. Dinapoli N, Casà C, Barbaro B et al (2016) Radiomics for rectal cancer. Transl Cancer Res 5:1-8

28. Ng F, Ganeshan B, Kozarski R et al (2013) Assessment of primary colorectal cancer heterogeneity by using whole-tumor texture analysis: contrast-enhanced CT texture as a biomarker of 5-year survival. Radiology 266:177-184

29. Karperien A, Ahammer H, Jelinek HF (2013) Quantitating the subtleties of microglial morphology with fractal analysis. Front Cell Neurosci 7:3

30. Captur G, Karperien A, Li C et al (2015) Fractal frontiers in cardiovascular magnetic resonance: towards clinical implementation. J Cardiovasc Magn Reson 17:80

31. Taylor JR (1997) An introduction to error analysis: the study of uncertainties in physical measurements. Cambridge University Press, Cambridge (UK)

32. Parmar C, Grossmann P, Bussink J et al (2015) Machine learning methods for quantitative radiomic biomarkers. Sci Rep 5:13087

33. Akaike H (1974) A new look at the statistical model identification. IEEE Trans Autom Control 19(6):716-723

34. International Commissioning on Radiation Units and Measurements.(2008) Receiver Operating Characteristic (ROC) Analysis in Medical Imaging. ICRU Report 79

35. Gonzalez RC, Woods E (2002) Digital image processing. PrenticeHall Inc, New Jersey

36. van Stiphout RG, Valentini V, Buijsen J et al (2014) Nomogram predicting response after chemoradiotherapy in rectal cancer using sequential PETCT imaging: a multicentric prospective study with external validation. Radiother Oncol 113(2):215-222

37. Intven M, Monninkhof EM, Reerink O et al (2015) Combined T2w volumetry, DW-MRI and DCE-MRI for response assessment after neo-adjuvant chemoradiation in locally advanced rectal cancer. Acta Oncol 54(10):1729-1736

\section{Affiliations}

\section{Davide Cusumano ${ }^{1,2} \cdot$ Nicola Dinapoli $^{3} \cdot$ Luca Boldrini $^{2} \cdot$ Giuditta Chiloiro $^{2} \cdot$ Roberto Gatta $^{3} \cdot$ Carlotta Masciocchi $^{2}$. Jacopo Lenkowicz ${ }^{2} \cdot$ Calogero Casà $^{3} \cdot$ Andrea Damiani $^{3} \cdot$ Luigi Azario $^{4} \cdot$ Johan Van Soest $^{5} \cdot$ Andre Dekker $^{5}$. Philippe Lambin ${ }^{5} \cdot$ Marco De Spirito $^{4} \cdot$ Vincenzo Valentini $^{2}$}

Davide Cusumano

davide.cusumano@policlinicogemelli.it
Nicola Dinapoli

nicola.dinapoli@policlinicogemelli.it 
Luca Boldrini

lucaboldrini@hotmail.it

Roberto Gatta

roberto.gatta.bs@gmail.com

Carlotta Masciocchi

carlotta.masciocchi@unicatt.it

Jacopo Lenkowicz

jacopo.lenkowicz@gmail.com

Calogero Casà

lillocasa@gmail.com

Andrea Damiani ad61965@gmail.com

Luigi Azario

luigi.azario@policlinicogemelli.it

Johan Van Soest

johan.vansoest@maastro.nl

Andre Dekker

andre.dekker@maastro.nl

Philippe Lambin

philippe.lambin@maastro.nl

Marco De Spirito

marco.despirito@policlinicogemelli.it
Vincenzo Valentini

vincenzo.valentini@unicatt.it

1 Polo scienze delle immagini, di laboratorio e infettivologiche, Università Cattolica del Sacro Cuore, Fondazione Policlinico Universitario Agostino Gemelli, Largo Francesco Vito 1, 00168 Rome, Italy

2 Polo Scienze Oncologiche ed Ematologiche, Istituto di Radiologia, Università Cattolica del Sacro Cuore, Fondazione Policlinico Universitario Agostino Gemelli, Largo Francesco Vito 1, 00168 Rome, Italy

3 Polo Scienze Oncologiche ed Ematologiche, Università Cattolica del Sacro Cuore, Fondazione Policlinico Universitario Agostino Gemelli, Largo Francesco Vito 1, 00168 Rome, Italy

4 Polo scienze delle immagini, di laboratorio e infettivologiche, Istituto di Fisica, Università Cattolica del Sacro Cuore, Fondazione Policlinico Universitario Agostino Gemelli, Largo Francesco Vito 1, 00168 Rome, Italy

5 Department of Radiation Oncology (MAASTRO), GROW School for Oncology and Developmental Biology, Maastricht University Medical Centre, Maastricht, The Netherlands 